\title{
THE HISTORY OF THE DEVELOPMENTS OF A VENEREAL DISEASE CLINIC *
}

By F. FOWLER WARD, M.B., B.Ch.

Medical Officer in charge of V.D. Department and Surgeon in charge of Urogenital Dept., East Suffolk and Ipswich Hospital.

Mr. Chairman, Ladies and Gentlemen,

In the first place I must thank the Council of the M.S.S.V.D. for the compliment they have paid this centre in holding the General Annual Meeting at Ipswich, and others for their presence this afternoon.

This being a provincial centre, and also not a teaching school, I cannot attempt to speak on recent or advanced clinical and pathological work, but shall confine my remarks to giving a brief description of the general routine, our difficulties and attempts at success: in short, a "Plain Man's Sermons." Hence the title, "The History of the Developments of a Venereal Disease Clinic." I hope they may be of some practical value to a budding V.D. Officer, and that criticism of others may be of value to myself.

In June, I9I9, I was appointed M.O. to a Clinic in embryo, and for the next six months studied Venereal Disease under the kindly direction of Drs. Clarkson and Malcolm Simpson at the London Hospital, and to both of these gentlemen I owe a great debt.

A definite method of treatment was necessary, and I have followed that of the London Hospital to a large extent ever since.

At the commencement, in January, I9I8, we had to make shift with what quarters we could get in the Outpatient Department with two Clinics per week, each Clinic being partly devoted to men, partly to women.

The Hospital and Local Authorities supported us loyally, but I have no doubt that we were looked on, by the Hospital generally, as an unmitigated nuisance, and when V.D. was mentioned, individuals, male and female, either held up their hands in pious horror or girded their loins and fled.

* Delivered before the Medical Society for the Study of Venereal Diseases at the Annual General Meeting at Ipswich, July 12, 1929. 


\section{HISTORY OF A VENEREAL DISEASE CLINIC}

The In-patient Department consisted of two rooms with four beds each on the first floor of what was known as the Septic Block.

The pathological work, i.e., culture for gonococci and Wassermann reactions, was done at the East Suffolk County Council laboratories, and examinations of smears for gonococci and sera for Spironema pallida by dark ground illumination by myself either during or at the end of the clinics. This immediate examination, I contend, is of great value, the patient receiving prompt attention and the Medical Officer learning to stand or fall by his own diagnosis.

A difficulty in the first years was that of getting suitable instruments, due to the War and partly also to one's own inexperience as to what were the necessary and really useful articles. I am glad to say that several things purchased in I9I7 are still in everyday use.

A further bane to the newly-elected M.O. was the visits of inspectors from the Ministry of Health. This was survived, however, and for some years their visits have been looked forward to as a means of exchanging views as to various methods of treatment, etc.

By the end of I9I8 the Clinic was fairly established. It had been a year of hard work, often very depressing, full of difficulties, which had to be met without a previous knowledge how to meet them. For instance, the Army Regulation D.40, under which a woman accused of infecting a soldier, was sent up for examination and report. Being an official under the Ministry of Health and not of the Army, and also being out of sympathy with the regulation, D.40 was not carried out at the Clinic; still, it was very worrying.

During the year I9I8 it was found that, with treatment of syphilitic patients, pregnant women and infants were mixed up with the "Profanum vulgum" to their detriment. Dr. John Adams was publishing his work at Thavies Inn and I went to see him, was received with courtesy, and inspired by his enthusiasm, a separate Clinic for children and their mothers was established in I9I9. It has always been interesting work, and I am indebted to Dr. John Adams for his kindly help.

A further matter arose. Coming to the Clinic were a large number of patients with non-venereal troubles. Being an O.P. Surgeon, it occurred to me to get hold of 


\section{BRITISH JOURNAL OF VENEREAL DISEASES}

these odds and ends, and so an O.P. Urogenital Clinic came into being. This is particularly useful in the treatment of strictures following gonorrhœa and other allied diseases.

Here, again, I am indebted to the kindness of others. Mr. Frank Kidd was holding a weekly clinic for cystoscopy, etc., at the London Hospital, and six months' work under him gave me an insight and predilection for it. There then came the question of material. There is a certain class of patient, chiefly men, known as Gonophobics. Cystoscopy gave them a mental satisfaction and certainly increased my knowledge of the normal bladder.

From its inception the work at the Clinic increased rapidly. Out-patient attendances for the year were :-

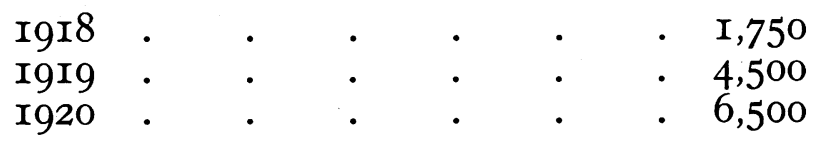

In fact, during I920 the amount of work taxed our full capabilities. The Wednesday evening Clinic, which nominally lasted from 4 p.m. to 7 p.m., was usually prolonged until 9.30 p.m. As far as Ipswich is concerned, this year was the peak owing to post-war conditions, and the figures for attendance soon dropped to, and have continued, between 4,000 and 5,000 per year.

After the War, and as a memorial, a scheme was started for new Hospital buildings, and we were promised a niche in them where the work could be carried on without being interrupted, and interrupting other people, and the existing department is the result.

Various causes were responsible for delay. In the year I920 it seemed as if we were going to be swamped with V.D. cases, and architectural plans were made accordingly, particularly for gonorrhœa in males, hence a rather elaborate irrigation room.

As I have stated, there were various delays between the planning of the buildings and their completion, and ideas of treatment had altered considerably. However, the V.D. Block was eventually completed and accepted by the Ministry of Health in I924-not without criticism-but I am still proud that the various rooms which had to be accommodated within the space allotted to us are 


\section{HISTORY OF A VENEREAL DISEASE CLINIC}

more or less according to my plans, guided, of course, by the architect.

The essential features are : an entrance from a quiet street, separate entrances, waiting and treatment rooms for both sexes, and more or less isolation from the Hospital as a whole.

Attached to the V.D. Block are two small wards for urogenital patients, also a convenience, as the same staff is utilised.

So much for a brief general survey, and now the present lines of treatment will be considered.

First of all the In-patient Department.

It has been found that early cases of uncomplicated gonorrhœa, both male and particularly female, clear up much more quickly when under skilled supervision than when attending as out-patients; hence some of these cases, where outside circumstances are bad, are admitted : Complications, such as rheumatism, iritis, acute prostatitis, severe secondary syphilis, buboes and others requiring surgical interference. It is an interesting fact that so far no case of operative pyosalpinx or salpingitis has been admitted into the V.D. wards : they seem to be the perquisite of the gynæcological or general surgical wards. Every new Resident Surgical Officer seems imbued with the idea that the V.D. beds are the ideal dumping ground for the unwanted tertiary syphilitic requiring in-patient treatment. He learns that the Ministry of Health does not agree with him. As far as a lumbar puncture for examination of cerebrospinal fluid is concerned, all cases, whether contagious or not, are admitted for one night.

\section{Out-patient Department}

At each Clinic patients are treated in rotation, thus syphilis, gonorrhœa, soft sore and non-venereal disease may all be seen during one session.

Soft sore is not often seen and may be disposed of at once.

Syphilis.-Before any treatment is given an attempt is made at an exact diagnosis, and the necessity of this is obvious. Thus, in addition to observing clinical symptoms, and taking blood for a Wassermann reaction, in every case where possible, serum is obtained for detection 


\section{BRITISH JOURNAL OF VENEREAL DISEASES}

of the Spironema pallida by dark ground illumination. The earlier and smaller the lesion the more is the prime importance of this method of examination; for the clinical evidence may be slight, the W.R. negative, and yet the patient may be suffering from syphilis.

It is difficult to say how soon a sore, which contains the Spironema, may develop after infection, but a few cases have come under my observation which would lead me to believe that it is sometimes a matter of three or four days only. Should one examination reveal no organism, further examinations are made in the course of the next few days, and in no case is an injection of arsenobenzol given on the off chance that the lesion is syphilitic.

If I have laid emphasis on this method of diagnosis it is because I attach the utmost importance to it. It is manifestly unfair to condemn a patient to a long course of treatment and mental anxiety when a microscopic examination may alter the diagnosis.

Again, the detection of the Spironema means that treatment is commenced at once and the patient may never become Wassermann positive.

Should the Spironema be found, blood is taken for the purpose of the W.R. and an injection of arsenobenzol given before the patient leaves the premises. Here, again, is the advantage of microscopic work being done by the Medical Officer, and the technique of dark ground illuminations once learned is easy and quick.

In the treatment of syphilis the " Ioo day" course, as advocated by Colonel Harrison, is chiefly used. The number of courses is governed by the W.R. at the end of each course and a further course is given after the W.R. has become negative, when, for some reason, such as Wassermann-fast cases or unsuitability of arsenobenzol, intra-muscular bismuth is substituted. Tests of cure are those suggested by the Ministry of Health in their Form V.21. An examination of the cerebrospinal fluid is made where possible. A few years ago cerebrospinal fluid was examined soon after the patients came for treatment, but it was found that if lumbar puncture was done early in the course of the disease the patient usually refused the second puncture required as a test of cure.

The actual preparation preferred is usually novarsenobillon. Stabilarson was used for some time, but a con- 


\section{HISTORY OF A VENEREAL' DISEASE CLINIC}

siderable number of immediate after-effects were noticed, also jaundice, and this latter drug has been dropped.

Very few serious complications have occurred from intensive arsenical treatment. This may be due to the fact that heroic doses are not given - the usual ones being 0.3 to $0.6 \mathrm{grm}$. It has been part of our policy to play for safety.

Being a provincial centre our doings are perhaps freely discussed by the laity, so I have preferred a middle course which, while giving adequate treatment, may not give startling results, but, on the other hand, does not cause violent accidents.

For arsenical dermatitis contramine was used; this, however, cost $7 s .6 d$. per dose, while ametox or Sod. thiosulphate is $6 d$. per dose. Being so impressed with the value of this drug in arsenical dermatitis, I have used it in other forms of dermatitis with equally good results. I do not try to explain its action.

Being, as it is, a centre for the treatment of Venereal Diseases, a considerable number of cases of tertiary syphilis come up. Although strictly outside the scope of the Ministerial regulations, these cases are treated as outpatients. The excuse, if any be needed, is that continuity of treatment is obtained to their benefit and with little extra cost, beyond the time of the Medical Officer. It is found that tabes is rendered non-progressive, and in many cases very definitely improved, by more or less regular courses of arsenobenzol and bismuth spread over a long period with the intervening administration of iodides or mercury. Similarly, syphilis of the heart and aorta, as evidenced by myocarditis and aneurysm, improve very greatly by careful administration of arsenobenzol and bismuth.

As regards the Children's Clinic, the cases seen are chiefly those of syphilis. A few cases of gonorrhœea in little girls occur. These are sent from the School Clinic and appear to be cases of accidental infection. The treatment consists of vaginal douche and bladder irrigation, followed later by swabbing with mercurochrome. It is surprising how soon children will allow treatment at the hands of the Sister when their mothers are excluded.

Similarly, we have very little trouble in giving intravenous injections to children. Patience, an occasional bribe of a penny, when a second veni puncture has to be 


\section{BRITISH JOURNAL OF VENEREAL DISEASES}

performed, does wonders and our children come up smiling. Infants, however, greet us with a hearty bawl, even before an intramuscular injection has been given.

Blood for the W.R. in infants is always obtained through the anterior fontanelle from the longitudinal sinus, but intravenous injections have never been given by this route-intramuscular medication only.

This Clinic was opened in IgIg and then galyl was used for some years with inunction of mercury.

The type of disease then obtaining in infants was severe. I remember some novelist describing a syphilitic babe as " a pot-bellied speckled toad." It was not a bad description.

A certain number of these infants died soon after treatment was instituted, not, I think, in consequence of it, but on their first appearance at the Clinic they were in a desperate condition. On the other hand, the cases that improved did so rapidly under treatment. As an evidence of this the numbers at the Children's Clinic increased very considerably, mothers recognising that their children were much better for treatment.

Thus, having got the children, the next step was to treat the mother. This followed without much difficulty ; the question of the fathers was, however, a different proposition, and I have not yet satisfactorily solved it-far otherwise. So at present we have a few mothers who come up for intensive arsenical treatment in the early months of each succeeding pregnancy; treatment is given whether the W.R. is positive or negative, and so far only one baby has been found positive.

Among the older children interstitial keratitis was fairly prevalent. At that time the idea seemed to prevail that arsenical treatment was of little value in this condition. It appeared to me that interstitial keratitis, being of syphilitic origin, ought to respond to antisyphilitic treatment, and in every case an intensive treatment, with intercurrent administration of mercury and iodide, was instituted, and this line of treatment is still being carried out.

The results were encouraging, the lesions cleared up fairly quickly - of course leaving scars-but very few recurrences are noted.

As evidence of the interest this Board of Governors takes in the work of the Hospital, I shall quote the follow- 


\section{HISTORY OF A VENEREAL DISEASE CLINIC}

ing case of congenital syphilis. In I 920 a boy of fourteen years old, an orphan, came under my care with advanced disease of the nasal bones and accessory sinuses, with such fœtid breath that it was difficult to know what to do with him ; apart from this defect he was a well-built, sturdy boy, but unemployable and dependent upon the Board of Guardians. W.R. was strongly positive ; intensive arsenical treatment was instituted, and, later, the aural surgeon took him in charge and cleaned out all dead bone, etc. He was still unemployable, having no nose, only a hole in the middle of his face. I made enquiries from Mr. Gillies, at Sidcup, and found that the boy could be taken in there if maintenance fees were guaranteed. The upshot was that through the influence of the Hospital Board, the Board of Guardians found the money and the boy returned in eighteen months with a good, if rather one-sided, nose, and eventually was found a job in Australia by the Salvation Army.

The type of syphilis in infants is different from that of ten years ago. Acute disease is seldom seen. When infants come, and they are few, it is usually snuffles or general malaise, no mucous patches of lips, mouth and genitalia or cachectic conditions due to advanced disease of the viscera. At present I have only one infant, W.R. +, who presented mild snuffles, a protuberant abdomen, and a history of not getting on. Weekly intramuscular bismuth, 0.05 grm., and inunction of Gordon's crème de mercure have improved his health.

Syphilis in children certainly does not appear so frequently, and we can only hope that it is our efforts in healing adults that have contributed to this result.

\section{GONORRHEA}

The larger part of the work in a mixed V.D. Clinic, such as this, is devoted to gonorrhœa, chiefly in the male.

The routine work consists in both sexes of irrigation of the urethra and bladder, and in the female of the vagina also. The best results certainly obtain where daily treatment is done by the Sister or male orderly. In the case of patients living in the borough, this daily treatment is the rule, and cuts short the course of the disease. Vaccine treatment with detoxicated vaccines was given for some 


\section{BRITISH JOURNAL OF VENEREAL DISEASES}

time, but, failing to get better results with it, vaccines have been given up. Tests of cure are those advocated by the Ministry of Health, and are: Urethral smear, three cultures of urine after prostatic massage, examination of prostatic bead, inhibition of alcohol, urethroscopy, or, where that is impossible, passage of a bougie, and instillation of nitrate of silver, $5 \mathrm{gr}$. to the ounce; to the urethra. All these tests must prove negative.

Among the complications of gonorrhoa, vesiculitis has interested me. For the chronic condition for some years I have performed Belfield's operation, i.e., vasotomy and injection of 20 per cent. argyrol, in cases where massage has failed. The most suitable cases are those in which the vesicles are dilated and on stripping them a considerable amount of débris is expelled on urination.

Lately Mr. Frank Kidd has advocated this method for recurrent acute posterior urethritis, in which the vesicles are involved, and has found it successful. Further and intractable complications depending on sepsis in the vesicles and prostate are rheumatism and iritis.

Quite recently a boy was admitted with severe iritis and a considerable amount of multiple arthritis occurring in the acute stage of gonorrhœa. The usual treatment caused no improvement, and the boy was ill and running an irregular temperature over the period of a fortnight. Under general anæsthesia both vasa were isolated and 5 to 7 c.c. of 20 per cent. argyrol were injected into each vesicle. The result was startling. Within twelve hours the severe iritis in both eyes had entirely cleared up, arthritis diminishing and general condition good. This improvement has been maintained, though there is still some slight antero-posterior urethritis.

An attempt was made this week to get a prostatic bead, but nothing was obtained, the vesicles were not tender or palpable.

This case suggests that vasotomy with injection of argyrol may be the treatment per se for iritis particularly and arthritis, and I shall certainly try it again.

Strictures, which usually occur when infectivity has disappeared, are now treated in the Urogenital Department, either by dilatation or urethrotomy, and are kept under observation for years.

One other point - the dealing with persons who cease 


\section{HISTORY OF A VENEREAL DISEASE CLINIC}

to attend. I have always thought that the only way to get patients to attend regularly is to take such personal steps as will impress them with the necessity of regular and continued attendance until such time as they are discharged cured.

Some patients require occasional reminders, and having a good intelligence department in the form of the Sister and orderly we can often get reminders conveyed to them. Writing letters to them, however, may cause trouble and vexation, both to the patient and the Medical Officer. It is sometimes necessary to look at the other person's point of view. 